\title{
Endophytic fungi from the roots of horseradish (Armoracia rusticana) and their interactions with the defensive metabolites of the glucosinolate - myrosinase - isothiocyanate system
}

Zsolt Szűcs ${ }^{1}$, Tamás Plaszkó ${ }^{1}$, Zoltán Cziáky ${ }^{5}$, Attila Kiss-Szikszai ${ }^{2}$, Tamás Emri ${ }^{3}$, Regina Bertóti ${ }^{4}$, László Tamás Sinka ${ }^{5}$, Gábor Vasas ${ }^{1}$ and Sándor Gonda ${ }^{1 *}$ (D)

\begin{abstract}
Background: The health of plants is heavily influenced by the intensively researched plant microbiome. The microbiome has to cope with the plant's defensive secondary metabolites to survive and develop, but studies that describe this interaction are rare. In the current study, we describe interactions of endophytic fungi with a widely researched chemical defense system, the glucosinolate - myrosinase - isothiocyanate system. The antifungal isothiocyanates are also of special interest because of their beneficial effects on human consumers.

Results: Seven endophytic fungi were isolated from horseradish roots (Armoracia rusticana), from the genera Fusarium, Macrophomina, Setophoma, Paraphoma and Oidiodendron. LC-ESI-MS analysis of the horseradish extract incubated with these fungi showed that six of seven strains could decompose different classes of glucosinolates. Aliphatic, aromatic, thiomethylalkyl and indolic glucosinolates were decomposed by different strains at different rates. SPME-GC-MS measurements showed that two strains released significant amounts of allyl isothiocyanate into the surrounding air, but allyl nitrile was not detected. The LC-ESI-MS analysis of many strains' media showed the presence of allyl isothiocyanate - glutathione conjugate during the decomposition of sinigrin. Four endophytic strains also accepted sinigrin as the sole carbon source. Isothiocyanates inhibited the growth of fungi at various concentrations, phenylethyl isothiocyanate was more potent than allyl isothiocyanate (mean $I C_{50}$ was 2.30-fold lower).

As a control group, ten soil fungi from the same soil were used. They decomposed glucosinolates with lower overall efficiency: six of ten strains had insignificant or weak activities and only three could use sinigrin as a carbon source. The soil fungi also showed lower AITC tolerance in the growth inhibition assay: the median I $C_{50}$ values were $0.1925 \mathrm{mM}$ for endophytes and $0.0899 \mathrm{mM}$ for soil fungi.
\end{abstract}

Conclusions: The host's glucosinolates can be used by the tested endophytic fungi as nutrients or to gain competitive advantage over less tolerant species. These activities were much less apparent among the soil fungi. This suggests that the endophytes show adaptation to the host plant's secondary metabolites and that host metabolite specific activities are enriched in the root microbiome. The results present background mechanisms enabling an understanding of how plants shape their microbiome.

Keywords: Myrosinase, Endophytes, Glucosinolate, Isothiocyanate, Fungal enzyme

\footnotetext{
* Correspondence: gondasandor@gmail.com;

gonda.sandor@science.unideb.hu

'Department of Botany, Division of Pharmacognosy, University of Debrecen,

Egyetem tér 1, Debrecen $\mathrm{H}-4010$, Hungary

Full list of author information is available at the end of the article
}

(c) The Author(s). 2018 Open Access This article is distributed under the terms of the Creative Commons Attribution 4.0 International License (http://creativecommons.org/licenses/by/4.0/), which permits unrestricted use, distribution, and

reproduction in any medium, provided you give appropriate credit to the original author(s) and the source, provide a link to the Creative Commons license, and indicate if changes were made. The Creative Commons Public Domain Dedication waiver (http://creativecommons.org/publicdomain/zero/1.0/) applies to the data made available in this article, unless otherwise stated. 


\section{Background}

It is now well established that the plant microbiome affects the health of plants, just as the much more researched human microbiome influences ours [1]. Of course, the complex groups of microbes also interact with the plant metabolites. Some natural products are co-products of the endophytes and the plants; several endophytes can selectively transform the plant's secondary metabolites [2]. Endophytic fungi are an intensively studied subset of the plant microbiome. A huge amount of scientific literature is gathering on this group, which is not that straightforward to define [3, 4]. Various definitions exist, but they are usually considered non-pathogenic strains that live inside plants without causing apparent symptoms [3, 4]. Endophytes are very good subjects for drug discovery. The possibility of finding new natural products is a significant driving force of the research of these fungi [5]. Endophytes are also well studied for production of different enzymes that operate under special conditions, for example, endophytes isolated from plants living in saline habitats, harbor enzymes that can operate at high salt concentrations [6]. Their beneficial interaction with plants also has potential uses in crop protection and plant health promotion (e.g. biocontrol, salt tolerance $[7,8])$. However, the nonpathogenic lifestyle can not be considered stable [3, 4]. "True" endophytes sometimes become latent pathogens, and this has severe agricultural impact $[9,10]$.
Despite all the above, the endophytes' interaction with the plant defense metabolites is not that studied.

The glucosinolate (GSL) - myrosinase (MYR) - isothiocyanate (ITC) chemical defense system is present in Brassicaceae, Capparaceae, Resedaceae and Moringaceae. It is one of the most researched plant defense systems [11]. Myrosinase (EC 3.2.1.147, thioglucoside glucohydrolase) catalyzes the reaction between a glucosinolate and water to yield glucose and a thiohydroximate. The free thiohydroximate subsequently spontaneously rearranges to isothiocyanates and byproducts (Fig. 1) [12, 13]. Most isothiocyanates are extremely pungent and exert various bioactivities due to their reactive -NCS groups, which results in strong antimicrobial, insecticidal and other effects [12]. This chemical defense system is present in vegetables of the Brassicaceae with various glucosinolates. Interestingly, the isothiocyanates have beneficial effects on humans, making the Brassicaceae crops healthy functional foods. Regular consumption of low amounts of isothiocyanates can prevent different human diseases [12].

Glucosinolates are chemically stable and are separated from their activating enzyme - MYR - by compartmentalization. They typically come in contact in planta when the plant tissue is damaged. There is a considerable variability of the actual separation strategy that depends on species, organ, developmental stage and many other factors [11]. Myrosinase and the GSLs are usually stored in separate cells, but this

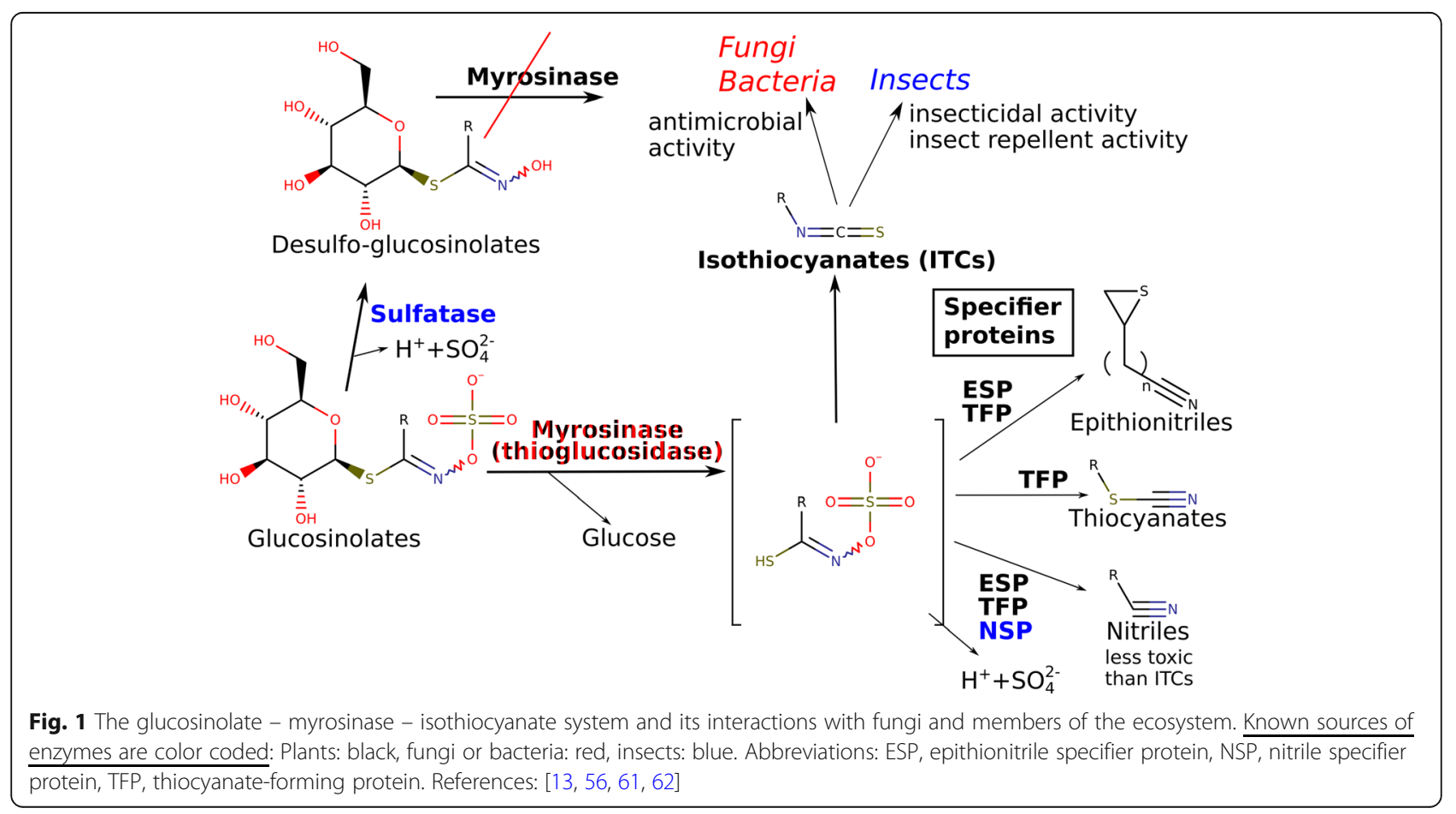


is not always the case. The GSL - ITC system is therefore considered a "chemical bomb" that is triggered by the attack of pathogens and herbivores [11]. The isothiocyanates were shown to have potent antifungal activity in many papers (e.g. [14-16]). It is an interesting question how endophytes tolerate the isothiocyanates of the plants. Actually, myrosinase activity can benefit microorganisms, as it enables them to obtain glucose from the thioglucoside. For endophytes, there would be an additional benefit as it would give them the option to disarm the "chemical bomb" of the host. Of course, they have to cope with the toxicity of the released ITCs or prevent its release (as shown for an Aspergillus sp. strain [17]). MYR activity was shown to be present in many microbial organisms including Aspergillus sp., Fusarium sp. [18], Citrobacter [19], Enterobacter [20] as well as many other species. Strains capable of metabolizing glucosinolates are part of the human gut microbial community [21-23].

Regarding effects of endophytes on the host's secondary metabolome, not many studies have been published to date. A study has shown that the endophytes specifically modify the plant metabolome to their own benefit. In particular, deglycosylation of flavonoid glycosides by the fungus Paraconiothyrium variabile was observed. The deglycosylated flavonoids displayed significant beneficial effects on the hyphal growth of germinated spores [24]. In addition, cases of selective in vitro modifications of plant secondary metabolites have been reported [2].

The effects of plant metabolites on endophytic fungi are examined in more detail. Studies suggest that chemical constituents actually drive the development of the microbiome around the plant roots. Microbes that can tolerate these metabolites are enriched in the rhizosphere. For example, Fusarium and Rhizopus species that can decompose the glucosinolate exudate were abundant in the soil around roots of Brassicaceae plants [25]. Some plant pathogens (e. g. Phoma lingam and Verticillium dahliae) can also decompose glucosinolates [26]. It is suggested that the decomposition of sinigrin is a detoxification process, as sinigrin hydrolysis had no effects on growth or spore germination of the tested fungi. Therefore, it was argued that the pathogens can perhaps use this ability to overcome the plant defenses. The pathogen Alternaria brassicicola adapts to the antifungal allyl isothiocyanate (AITC) via increased transcription of oxidative stress response genes. These include glutathione S-transferases, thioredoxins, glutathione peroxidases, $\gamma$-glutamylcysteine synthetases [27].

Though there are many reports about fungi with myrosinase activity, the community of endophytic fungi from Brassicaceae plants were not tested for myrosinase. As the GSL - MYR - ITC system is usually considered a chemical defense system against fungi, the presence of this activity would make this system more complex. Also, in such an approach, a direct comparison with some control group of fungi would be desirable so that the specificity of the phenomenon can be assessed.

The aim of the current study was to test the hypothesis that endophytes interfere with the GSL - MYR ITC chemical defense system of plants. The main questions are: 1., Do endophytes tolerate the ITCs of the host better than non-endophytic fungi? 2., Can endophytes decompose GSLs? Can they use them as a carbon source? 3., How specific is the activity of the endophytes? To answer the above questions, we used isolated fungal root endophytes from Armoracia rusticana (horseradish), a Brassicaceae crop with a very high abundance of ITCs. The strains were grown on horseradish extract to test their glucosinolate decomposing capacity and their ability to produce various decomposition products. Their ITC tolerance and ability to use GSLs as a sole carbon source was also examined. As a reference group, a set of soil fungi from the same soil was used.

\section{Methods \\ Chemicals}

Reagents were of analytical purity. Media components (peptone, glucose monohydrate, sodium nitrate, potassium hydrogenphosphate, magnesium sulfate, potassium chloride, ferrous sulfate) were from Reanal (Budapest, Hungary). Allyl isothiocyanate, allyl cyanide, 2-phenylethyl isothiocyanate and phenylpropionitrile and glutathione (reduced form) were from Sigma Aldrich (MO, USA). Pure glucosinolates were from Phytoplan (Germany). LCMS grade acetonitrile, water and formic acid were purchased from Fisher Scientific (Belgium). Solvents (1-propanol, 2-propanol) and media additives (streptomycin, chloramphenicol and dichloran) were from VWR. Double distilled water was used throughout the study.

\section{Standard microbiological media}

The following media and compositions were used: Saboraud Glucose agar broth or agar (SGB or SGA, $40 \mathrm{~g} / \mathrm{L}$ glucose monohydrate, peptone $10 \mathrm{~g} / \mathrm{L}, \mathrm{pH} 5.6 \pm 0.2$ ); potato dextrose agar (PDA, potato infusion equivalent to $200 \mathrm{~g} / \mathrm{L}, 20 \mathrm{~g} / \mathrm{L}$ glucose monohydrate, $\mathrm{pH} 5.6 \pm 0.2$ ); Czapek-Dox medium (CzD, 3.0 g; $\mathrm{NaNO}_{3} ; 1.0 \mathrm{~g} \mathrm{~K}_{2} \mathrm{HPO}_{4}$; $0.5 \mathrm{~g} \mathrm{MgSO}_{4} \cdot 7 \mathrm{H}_{2} \mathrm{O} ; 0.5 \mathrm{~g} \mathrm{KCl}$ and $0.01 \mathrm{~g} \mathrm{FeSO}_{4} \cdot 7 \mathrm{H}_{2} \mathrm{O} / \mathrm{L}$, $\mathrm{pH} 7.3 \pm 0.2$ ). Media were solidified with $2 \%$ agar, where appropriate.

\section{Isolation of endophytes}

Endophytic fungi from horseradish (Armoracia rusticana) were used for the study. Healthy horseradish roots from the cultivated populations, from the region of Ujléta or Debrecen were used. Collection of the roots complied with local legislation and guidelines. 
Horseradish roots were surface-sterilized with 4-fold diluted commercial bleaching solution, followed by several rinses with sterile distilled water. Afterwards, the roots were cut into pieces (approximately $2 \times 2 \times 1 \mathrm{~cm}$ in size) and placed on SGA for endophyte isolation. Surface sterility was checked by imprinting the surface-sterilized plant tissue onto SGA plates, and/or spreading aliquots of the last rinse fluid on the same type of medium. When no growth in these negative control plates was observed, the explant batch was considered free of surface contaminants, as proposed by [28]. The plates were sealed and stored at room temperature. The fungi appeared at the edge of the explants were considered endophytes. The isolated fungi are referred to as E1-E7 throughout the manuscript.

\section{Isolation of soil fungi}

For comparison with the endophytes, a set of soil fungi were obtained from the Debrecen site, where some of the horseradish roots were from. Sampling was performed in June of 2016. No plants were present at the sampling point. About $1 \mathrm{~g}$ of soil and $10 \mathrm{~mL}$ of sterile water were mixed vigorously to form a suspension. After $10^{3}$-fold dilution of the above stock suspension, $100 \mu \mathrm{L}$ of the diluted soil suspension was spread onto PDA or SGA plates containing $50 \mathrm{mg} \mathrm{L}^{-1}$ streptomycin and $50 \mathrm{mg} \mathrm{L}^{-1}$ chloramphenicol. A subset of isolation plates also contained $2 \mathrm{mg} \mathrm{L}^{-1}$ dichlorane. The appeared fungi were subcultured to obtain pure strains and used for the later study. Of these soil fungi, 10 apparently different strains were randomly selected for the current work. These are referred to as S1-S10 throughout the manuscript.

\section{Identification of strains}

Pure strains were grown in shaken cultures, lyophilized, and their DNA was purified for identification of species. DNA sequencing was carried out as described earlier [29] using the following primer pairs: ITS1, ITS4, (ITS); Bt2a, Bt2b ( $\beta$-tubulin); act-512F, ACT-783R ( $\alpha$-actin) and EF1728F, EF1-986R (translation elongation factor $1 \alpha$ ). The obtained sequences were deposited at the GenBank nucleotide sequence database under the following accession numbers: KP191628-KP191640. Isolates were identified by comparison of their sequences with sequences available at GenBank (http://www.ncbi.nlm.nih.gov/genbank), Q-bank Fungi (http://www.q-bank.eu/fungi/) and Fusarium ID (http://isolate.fusariumdb.org) databases.

\section{Preparation of horseradish root extract (HRE)}

To examine the fungal decomposition ability against different glucosinolates, a growth medium from the host plant was prepared and used for the study. This aqueous liquid contained the metabolome of the horseradish at approximately original concentration. About 500-1000 g of healthy horseradish roots were cut into large pieces and cooked in water for $30 \mathrm{~min}$ to inactivate the myrosinase. Thereafter, the cooked roots were homogenized with $\mathrm{MeOH}$ in a 3:2 solvent to fresh weight ratio and subsequently boiled $\left(80^{\circ} \mathrm{C}\right)$ under reflux for $30 \mathrm{~min}$. The extract was filtered and evaporated to dryness in a rotary evaporator. Then, the dry residue was dissolved in water corresponding to the original water content of the roots $(70 \%$ water content is typical). The liquid was filtered sterile on $0.20 \mu \mathrm{m}$ PES membranes after a series of prefiltrations, and was stored at $-24{ }^{\circ} \mathrm{C}$ before use. If necessary, the liquid was supplemented with $2 \%$ autoclaved agar.

\section{Preparation of fungal inoculums}

For the initiation of experiments, liquid suspensions of the fungi were used to ensure uniform inoculation of wells and samples. Fungi were grown in $30 \mathrm{~mL}$ of either $2 \%$ malt extract broth, Czapek Dox medium or HRE in shaken cultures at room temperature, $200 \mathrm{rpm}$. The time necessary for full growth was usually 3-9 days. Sterile glass homogenizers were applied to disintegrate the washed fungal mycelia without the use of quartz sand. The suspensions of the fungal inoculums were stored at $4{ }^{\circ} \mathrm{C}$ before the experiments. As the species differed in morphology and the ability to form conidia, dry weight per $\mathrm{mL}$ was chosen as a means of standardization. Dry weight was determined gravimetrically after lyophilization of the inoculum suspensions.

\section{Incubation of horseradish extract with fungi}

The fungi were incubated in the previously described horseradish extract to detect their effects on the horseradish secondary metabolites. Five $\mathrm{mL}$ aliquots of HRE were inoculated with fungal inoculums equivalent to $40 \mu \mathrm{g}$ dry weight per $\mathrm{mL}$. Thereafter, to correct for dilution, the liquid was separated into test tubes so that each tube contained an amount equivalent to $250 \mu \mathrm{L}$ HRE. The tubes were placed in a sterile plastic container where sterile bidistilled water provided high relative humidity to minimize evaporation during the study. Samples were frozen and stored at $-24{ }^{\circ} \mathrm{C}$ until further processing. At least 3 replicates were collected for each data point. Control was not inoculated. The experiment was run for 16 days. Sampling times were based on preliminary experiments. To provide samples at the different points of the growth curves, the seven endophytic strains were sampled at different days. A similar experiment was run with the soil fungi, in this case, only the 16-day samples were processed. After lyophilization of the samples, the samples were dissolved in $250 \mu \mathrm{L} 10 \%$ $1-\mathrm{PrOH}$ to help centrifugation and reduce the dissolution of viscous polysaccharides. The fungal pellet was liophilized and the dry mass was measured to obtain the growth curve of the different strains. 
Measurement of glucosinolate decomposition (LC-ESI-MS) The glucosinolate decomposition by endophytic and soil fungi was quantified / screened in LC-ESI-MS. The UHPLC system (Dionex Ultimate 3000RS) was coupled to a Thermo Q Exactive Orbitrap mass spectrometer (Thermo Fisher Scientific Inc., Waltham, USA) equipped with an electrospray ionization source (ESI). The column was a Phenomenex Kinetex XB- $\mathrm{C}_{18}$ column $(100 \mathrm{~mm} \times 2$. $1 \mathrm{~mm} \times 2.6 \mu \mathrm{m})$. Oven temperature was maintained at $30{ }^{\circ} \mathrm{C}$; the flow rate was $250 \mu \mathrm{L} \mathrm{min}{ }^{-1}$. Eluent $\mathrm{A}$ was water and eluent B was acetonitrile, both contained $0.1 \%$ formic acid. The following gradient elution program was used: 0 min, $5 \%$ B, 0-2 min, $5 \%$ B; $2-5$ min, $25 \%$ B; 56 min, $60 \%$ B; 6-7 min, $100 \%$ B; 7-9 min, $100 \%$ B; 9$10 \mathrm{~min}, 5 \% \mathrm{~B}, 10-18 \mathrm{~min}, 5 \% \mathrm{~B}$. One $\mu \mathrm{L}$ of the 200 -fold dilutions (with $\mathrm{MeOH}$ ) of the redissolved HRE samples was injected in every run. With a serial dilution, it was shown that this dilution is within the linear range of the analytes of interest. The Q Exactive hybrid quadrupoleorbitrap mass spectrometer operated in negative ion mode with the following parameters: capillary temperature $320^{\circ}$ C, spray voltage $3.8 \mathrm{kV}$, the resolution was set to 70,000 . The mass range scanned was $150-1000 \mathrm{~m} / \mathrm{z}$. The maximum injection time was $100 \mathrm{~ms}$. Sheath gas and aux flow rates were 32 and 7 arb, respectively. The raw data were processed by the XCMS Online platform [30], using parameters suggested for the Orbitrap instrument, with minor modifications. The peak lists were further processed in R [31], using ggplot2 [32]. For quantification of major GSLs, five-point calibration curves were constructed using sinigrin and gluconasturtiin. The calibration curve spanned the concentration range of 0.01$5 \mu \mathrm{g} \mathrm{mL} \mathrm{L}^{-1}$. For the quantification of GSH, a calibration curve in the range of $0.01-5 \mu \mathrm{g} \mathrm{mL}^{-1}$ was used. For GSHAITC quantification, the derivatization reaction was adapted from our recent study [33]. A five point calibration curve was constructed by reacting $0.001-1 \mu \mathrm{g} \mathrm{mL} \mathrm{L}^{-1}$ AITC with excess GSH at pH 7.5, in $\mathrm{NH}_{4} \mathrm{OAc}$ buffer [33].

\section{SPME-GC-MS of microbial volatile organic constituents}

Typical voltatile glucosinolate decomposition products (isothiocyanates and nitriles) were sought by a newly developed SPME-GC-MS method. Ten $\mathrm{mL}$ of horseradish extract with $2 \%$ agar was poured in Petri dishes $9 \mathrm{~cm}$ in diameter. This amount contains approximately $60 \mu \mathrm{mol}$ sinigrin. The plate was inoculated with $100 \mu \mathrm{L}$ aliquots of fungal inoculums that were spread on the surface of the medium. The cultures were grown upside down. Five $\mathrm{mg}$ of activated charcoal was placed in sterile tube caps in the lid of the Petri dishes to adsorb the volatile constituents from the air above the fungal culture. The sorbents were replaced daily. The charcoal samples were stored at $-24{ }^{\circ} \mathrm{C}$ before analysis. Elution from the charcoal was accomplished by methyl acetate and the resulting liquid was directly injected into GC-MS. Some additional technical details and the steps of this optimization are given in the Additional file. Measurement parameters were as follows: split ratio was 10:1; inlet temperature was $150{ }^{\circ} \mathrm{C}$. Time program: Initial oven temperature was $35{ }^{\circ} \mathrm{C}$, held for $3 \mathrm{~min}$, followed by a $15{ }^{\circ} \mathrm{C} /$ min temperature gradient to $90{ }^{\circ} \mathrm{C}$, then $50{ }^{\circ} \mathrm{C} /$ min to $320^{\circ} \mathrm{C}$ and held for $2 \mathrm{~min}$. A HP-5MS 5\% Phenyl Methyl Siloxane column was used. Carrier gas was He;

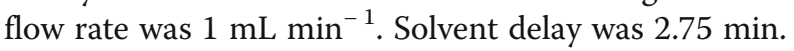

As five-point calibration curves, serial dilutions of authentic standards of allyl cyanide, allyl isothiocyanate, phenylpropionitrile and 2-phenylethyl isothiocyanate were used.

\section{Glucosinolate sinigrin as a sole carbon source for fungal growth}

In this experiment, the growth of the fungal strains on a synthetic medium was tested. A plant defensive metabolite, sinigrin was added as the sole carbon source. The inoculum volume was minimized to limit the amount of nutrient carryover. The growth medium was Czapek Dox medium with sinigrin, added at a concentration equimolar to $2 \%$ sucrose. As a positive control, the same medium with $2 \%$ glucose was used. Water served as negative control. The experiment was run in 96-well plates for 12 days. Hundred $\mu \mathrm{l}$ of medium were added to each well. All media were sterile filtered using PES filters. The fungal growth was tested by measuring the increase in absorbance by a plate reader at $800 \mathrm{~nm}$. The changes were confirmed by visual investigation. Growth was tested in four replicates.

\section{Determination of $\mathrm{IC}_{50}$ values for growth inhibition of fungi by allyl isothiocyanate and 2-phenylethyl isothiocyanate}

The growth inhibitory effect of the plant defensive compounds were investigated on the fungi. The endophytes and the soil fungi were directly compared. Aliquots of Saboraud Glucose Broth (SGB) media $(500 \mu \mathrm{L})$ were inoculated with fungi in 24-well plates. Growth was monitored by a plate reader at $800 \mathrm{~nm}$, using $5 \times 5$ points of absorbance determination evenly distributed within the well, thereby allowing quantification of growth of filamentous fungi. When growth started and an increase in absorbance was found, the cultures were treated with $5 \mu \mathrm{L}$ aliqouts of diluted AITC or PEITC. Serial dilution was performed with DMSO. As a preliminary study, final concentrations of both AITC and PEITC were 0.25, 2.5, 25, $250 \mu \mathrm{g} \mathrm{mL}^{-1}$. A $2500 \mu \mathrm{g} \mathrm{mL}^{-1}$ equivalent treatment was also tested. In this case, the final concentration is not accurate as the medium becomes saturated with AITC or PEITC. A later experiment added several other concentrations if resolution was insufficient to 
determine an $\mathrm{IC}_{50}(0.445,0.791,1.406,4.45,7.91,14.1$, 44.5, 79.1, 141, 445, 791, $1406 \mu \mathrm{g} \mathrm{mL} \mathrm{m}^{-1}$ equivalent). Control was treated with DMSO. As the ITCs are volatile to some extent, a single plate was treated with a single concentration of an agent. Different fungi were therefore allowed to grow 12, 24 or $36 \mathrm{~h}$ before treating the whole plate at the same time. The treated plates were incubated in darkness, at room temperature for $48 \mathrm{~h}$. Growth was calculated by subtracting the zero time absorbance (measured just before treatment) from the absorbance measured at $48 \mathrm{~h}$ after treatment. Each point subjected to an $\mathrm{IC}_{50}$ calculation in $\mathrm{R}$ (using 'drc' [34]) was a mean of at least 3 replicates.

\section{Statistical analyses}

To determine whether a concentration of a metabolite significantly changes as a result of treatments, ANOVAs were run on the features independently using XCMS online [30]. In XCMS Online, each point of observation was subjected to ANOVA as a separate treatment. A point of observation consisted of the samples of a treatment at a given time, $n \geq 3$. As many statistical hypotheses were tested, Bonferroni correction was used for these data; features were significant at $p<3.03 \mathrm{e}-6$. After successful ANOVAs, Dunett's test was used as a posthoc test for comparison of multiple treatments to the controls. Either end-point data or data points with nearest control treatment points were compared (both $n=$ 3). All calculations were performed in R [31].

\section{Results and discussion Endophytic fungi}

Seven endophytic fungi were isolated from surfacesterilized root segments of Armoracia rusticana. Identification showed that the isolated endophytes belong to different taxonomic groups. The isolated strains were purified and subjected to identification via sequencing taxonomically relevant segments of their DNA (ITS, $\beta$ tubulin, $\alpha$-actin and translation elongation factor $1 \alpha$ ). By comparing the sequences with sequences available at GenBank, the isolates were found to belong to the species shown in Table 1. The set contains members of three taxonomic-functional groups: The two Fusarium oxysporum isolates $(E 1, E 3)$ belong to the Hypocerales. This species was shown to have endophytic strains in a relative plant species, Brassica napus [35]. The members of the other group belong to the related clades Pleosporales (E46) and Botryosphaeriales (E2) [36]. These species have also been described as endophytes [37, 38]. The finding of Oidiodendron cerealis (E7) is somewhat unusual, as it is a typical Ericaceous ("ericoid") endophyte [39], which favors hosts from the Ericaceae plant family [40].

\section{Glucosinolate decomposition by horseradish endophytes} and soil fungi.

Several tested fungi (Macrophomina phaseolina, Fusarium oxysporum, Setophoma terrestris, Paraphoma radicina) were shown to decompose various glucosinolates; the endophytes were overall more efficient decomposers than the soil fungi from the same soil. The rates of decomposition depended on the side chain of the glucosinolates to some extent, as detailed later. The main method of this approach was incubation of the fungal strains in horseradish extract (HRE) as a growth medium, and quantification of glucosinolate concentrations by LC-MS. This allowed the testing of the fungi for putative myrosinase activity, but required purified strains of endophytes and soil fungi.

Various glucosinolates could be identified from the horseradish extract. LC-MS/MS examination showed that a $250 \mu \mathrm{L}$ aliquot (equivalent to $0.295 \mathrm{~g}$ horseradish FW or 0 . $088 \mathrm{~g}$ horseradish DW) contained $1.42 \mu \mathrm{mol}$ sinigrin (2. $37 \mathrm{mg} \mathrm{mL}{ }^{-1}$ ), $0.077 \mu \mathrm{mol}$ gluconasturtiin (2-phenylethyl glucosinolate) (129 $\left.\mu \mathrm{g} \mathrm{mL} \mathrm{m}^{-1}\right)$ and several, less abundant minor GSLs (Table 2). The LC-MS dataset of the incubation was subjected to automatic feature detection by XCMS Online, followed by targeted screening for glucosinolates. If a feature had chromatographic peak shape and it yielded at least two of the GSL-specific fragments in MS/MS, it was identified as a glucosinolate. For this purpose, we used the characteristic ions $259.012\left(\mathrm{C}_{6} \mathrm{H}_{11} \mathrm{SO}_{9}^{-}\right)$and 96.960 $\left(\mathrm{HSO}_{4}^{-}\right)$in $\mathrm{MS}^{2}[41,42]$. The detected minor glucosinolates included aliphatic (gluconapin, glucocochlearin), thiomethylalkyl (glucoiberin), and indolic (glucobrassicin) types as well (Table 2, Additional file 1: Figure S1). Agneta et al. has previously reported these glucosinolates in horseradish roots [43]. Though the detection sensitivity of GSLs by LC-ESI-MS is variable to some extent, several of the detected GSLs have a response ratio between $0.73-1.72$ vs sinigrin $[44,45]$. Therefore, the minor GSLs are estimated to be present at quantities between $0.7-40.6 \mathrm{nmol}$ (each) in a $250 \mu \mathrm{L}$ aliquot.

In controls, the concentrations of most glucosinolates did not change significantly during the 16 day incubation period $(p>0.05)$. Six out of seven endophytic fungi successfully decomposed most or all glucosinolates $(p>$ 0.05, Fig. 2, Additional file 2: Table S1). By the end of incubation, six of seven strains significantly decreased the amount of the major glucosinolate, sinigrin $(p<0.001$, Fig. 2a). However, strain $E 7$ (O. cerealis) could not decompose any glucosinolates (Fig. 2, Additional file 1: Figure S2). The sinigrin decomposition ability was also tested for the same fungi in Saboraud glucose broth spiked with a similar amount of sinigrin. However, replacing the horseradish extract medium resulted in several false negative results for sinigrin decomposition with the same endophytic fungi (Additional file 1: Figure 
Table 1 List of endophytes identified in horseradish roots

\begin{tabular}{llll}
\hline ID & Species & Sequenced gene(s) & GenBank accession numbers \\
\hline E1 & Fusarium oxysporum species complex & ITS, eF1 & KP191628, KP191635 \\
E2 & Macrophomina phaseolina & ITS, $\beta$-tubulin & KP191629, KP191637 \\
E3 & Fusarium oxysporum species complex & ITS, eF1 & KP191630, KP191636 \\
E4 & Setophoma terrestris & ITS, a-actin & KP191631, KP191638 \\
E5 & Paraphoma radicina & ITS, a-actin & KP191632, KP191639 \\
E6 & Paraphoma radicina & ITS, a-actin & KP191633, KP191640 \\
E7 & Oidiodendron cerealis & ITS & KP191634 \\
\hline
\end{tabular}

S3). This suggests that the presence of high amounts of GSLs in is not sufficient alone to induce enzymes that are responsible for the breakdown, despite the inherent ability to degrade glucosinolates is there (E2, E4-E6) (Fig. 2 vs Additional file 1: Figure S3). This underlines the importance of working in HRE to show the decomposition ability of the fungi. Despite the composition of HRE is an average of the different types of cells in the horseradish root, it resembles the in planta conditions better than a standard microbiological medium. Using whole plant extracts was also shown to be superior to endophyte studies by an other group in terms of the number of endophyte emergents as well as the growth character of the endophye cultures [46]. When nonmyrosin cells are intracellularly penetrated by endophytes, the fungal myrosinase enzymes can decompose the glucosinolates therein. These enzymes can either be extracellular or intracellular [47].

After calculating the slope of concentration decrease for SGN and GLN, decomposition rates span the range $0.606-1.476 \mathrm{mM} \mathrm{d}-1$ and $0.018-0.057 \mathrm{mM} \mathrm{d}^{-1}$ respectively (Table 3 ). Within-species differences are evident for strains $E 1-E 3$ (F. oxysporum) and E5 E6 ( $P$. radicina). While E1 rapidly decomposed most glucosinolates already during its growth stage (1.
$125 \mathrm{mM} \quad \mathrm{d}^{-1}$ SGN; $\left.0.057 \quad \mathrm{mM}^{-1} \quad \mathrm{GLN}\right), \quad$ E3 performed a much slower degradation only after its growth has stopped $\left(0.606 \mathrm{mM} \mathrm{d}^{-1}\right.$ SGN; $0.030 \mathrm{mM}$ $\mathrm{d}^{-1}$ GLN) (Additional file 1: Figure S4). The same strains differed much less in the case of indolic glucosinolates (Fig. 2d, Additional file 1: Figure S2i-j). The differences between strains E5 and E6 were less pronounced when compared to the differences between strains E1 and E3. Strains E5 and E6 both strains reached the stationary phase of growth at around day 9-10 (Additional file 1: Figure S4), but strain E5 started decomposition of glucosinolates a few days earlier. Once the decomposition of SGN started, it ran with similar rate in the media of E5 and E6 (Table 3). The difference was more prominent for methylthioalkyl glucosinolates (Fig. 2b, Additional file 1: Figure S2e-f) and aromatic glucosinolates (Fig. 2c, Additional file 1: Figure S2 g-h). By the end of the study, E6 decreased the concentration of thiomethylalkyl glucosinolates only, but the decrease was not significant $(p>0.05)$, in contrary to E5 $(p<0.001)$. On the other hand, E6 decomposed gluconasturtiin at 1.72-fold speed compared to $E 5$ [Table 3]. Strain E4 showed a behavior similar to E5-E6, especially for methylthioalkyl and aromatic GSLs (Fig. 2b-

Table 2 Identified glucosinolates found in the horseradish extract. These compounds were subjected to fungal decomposition by horseradish endophytes and soil fungi

\begin{tabular}{lllll}
\hline$[\mathrm{M}-\mathrm{H}]^{-}(\mathrm{m} / \mathrm{z})$ & Rt & Common name & Side chain & Glucosinolate class \\
\hline 358.0275 & 1.59 & Sinigrin* & 2-propenyl & aliphatic \\
372.0434 & 2.69 & Gluconapin & 3-butenyl & aliphatic \\
374.0591 & 4.23 & Glucocochlearin & 1-methylpropyl & aliphatic \\
386.0592 & 6.37 & Glucobrassicanapin & 4-pentenyl & aliphatic \\
422.0261 & 1.28 & Glucoiberin* & 3-(methylsulfinyl)propyl & methylthioalkyl \\
478.0888 & 7.40 & Glucoibarin & 7-(methylsulfinyl)heptyl & methylthioalkyl \\
408.0435 & 7.32 & Glucotropaeolin & benzyl & aromatic \\
422.0591 & 9.08 & Gluconasturtiin* & phenylethyl & aromatic \\
447.0544 & 8.49 & Glucobrassicin* & indol-3-ylmethyl & indole \\
477.0649 & 9.29 & 4-Methoxyglucobrassicin & 4-methoxyindol-3-ylmethyl & indole \\
\hline
\end{tabular}

Glucosinolates whose identity is also proven by comparison to an authentic standard are marked with an asterisk, the rest is identified from the results of [43] Abbreviation: Rt retention time (min) 

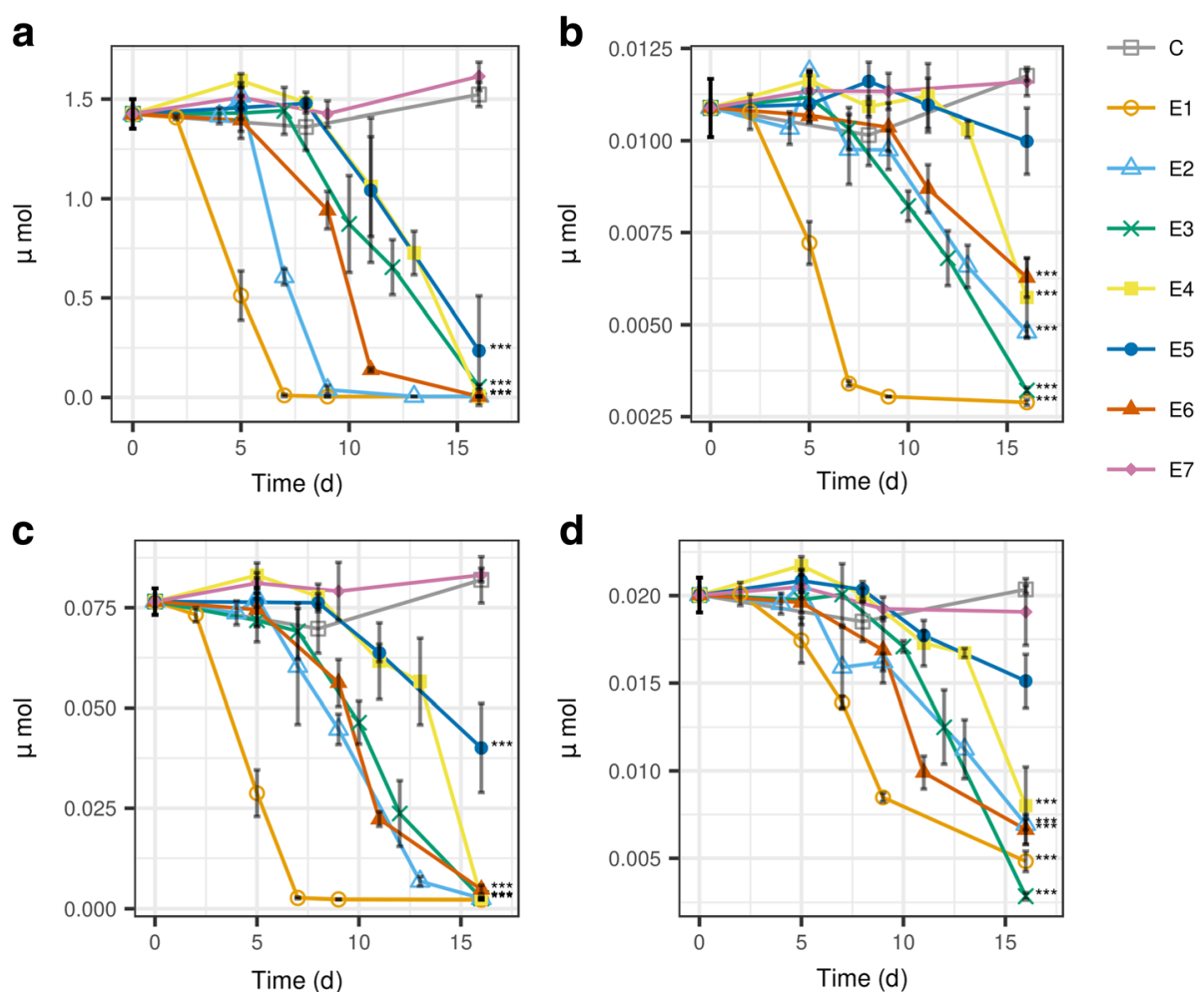

Fig. 2 Decomposition of glucosinolates in horseradish extract inoculated by endophytic fungi from horseradish. Subplots: a., sinigrin; b., glucoiberin; c., gluconasturtiin; d., glucobrassicin. Fungi: E1, Fusarium oxysporum; E2, Macrophomina phaseolina; E3, Fusarium oxysporum; E4, Setophoma terrestris; E5, Paraphoma radicina; E6, Paraphoma radicina; E7, Oidiodendron cerealis; C, control (not inoculated). Statistical test: Dunnett's test, endtime samples compared to end-time control $\left(n=3,{ }^{* *}, p<10^{-5} ; *^{* *}, p<10^{-4 *}, p<5^{*} 10^{-4}\right)$. For the data in Table form, see Additional file 1: Table S1

c, Additional file 1: Figure S2e-h). It decomposed GSLs only after reaching the stationary phase of development. Strain E2 was much more effective in the decomposition of aliphatic GSLs than the slow growing strains E4-6 (Additional file 1: Figure S2b-d). However, these fungi degraded aromatic and methylthioalkyl GSLs with similar efficacy (Fig. 2b-c, Additional file 1: Figure S2e-h).

The differences between decomposition rates of different classes of glucosinolates is also apparent from these data. The indolic GSLs were shown to be less prone to fungal decomposition (Fig. 2d, Additional file 1: Figure $\mathrm{S} 2 \mathrm{i}-\mathrm{j})$, even though they were present at much lower concentrations than the main compounds. The other classes (aliphatic, methythiolalkyl and aromatic) were decomposed with more or less similar efficacy.

The glucosinolate decomposing ability was less prevalent in the tested set of soil fungi. After the same 16-day incubation and sample preparation, the HRE samples were subjected to LC-MS for glucosinolate screening. The results showed that while some soil fungi were active, their overall efficacy was lower than that of the endophytes (Fig. 3, Additional file 1: Figure S5, Table 3, Additional file 2: Table S2). Four of ten strains decomposed all sinigrin by the end of the incubation period $(S 1-2, S 9-10)$; two were only weak decomposers $(S 4, S 6)$ and four could not metabolize sinigrin $(S 3, S 5, S 7-8)$.

Decomposition of other aliphatic GSLs was somewhat less efficient (Additional file 1: Fig. 5c-d, Table 3, Additional file 2: Table S2). Despite being able to decompose sinigrin and aliphatic GSLs, S1 could not efficiently decompose indolic GSLs (Fig. 3d, Additional file 1: Figure S5i-j, Additional file 2: Table S2). This is similar to the phenomena observed in the case of endophytes. The active soil fungi, on the other hand, showed somewhat higher decomposition rates than endophytes (Table 3).

Thioglucosidase/myrosinase enzymes of fungal origin have already been described before [17, 18, 26, 47] Based on our results, it seems, that this enzymatic activity is quite widespread. The potential glucosinolate decomposing ability shown above is a fine example of how the microbial community can modify the plant metabolome in various ways. The results also highlight the importance of within-species variability when studying plant - microbe interactions, as also shown by [48]. The fate of the glucosinolates was unclear at this point, therefore we aimed to detect their volatile decomposition products by SPME-GC-MS. 
Table 3 Summary of the tested parameters of endophytic fungi isolated from horseradish and soil fungi

\begin{tabular}{|c|c|c|c|c|c|c|c|c|c|c|c|}
\hline \multirow[b]{2}{*}{ ID } & \multirow{2}{*}{$\begin{array}{l}\text { Sole } C \text { source }^{a} \\
\text { SGN }\end{array}$} & \multicolumn{4}{|c|}{$\begin{array}{l}\text { Glucosinolate decomposition in horseradish extract } \\
\text { Residual amount after } 16 \mathrm{~d}(\%)^{\mathrm{b}}\end{array}$} & \multicolumn{4}{|c|}{$\begin{array}{l}\text { Decomposition rate in horseradish extract } \\
\left(\mathrm{mM} \mathrm{d}^{-1}\right)\end{array}$} & \multicolumn{2}{|c|}{$\begin{array}{l}I_{50} \text { liquid culture } \\
(\mathrm{mM})\end{array}$} \\
\hline & & SGN & GIB & GLN & GBR & SGN & GIB & GLN & GBR & AITC & PEITC \\
\hline$\overline{E 1}$ & + & $0.2 \pm 0 \%$ & $3.7 \pm 0.9 \%$ & $0 \pm 0 \%$ & $17 \pm 3.1 \%$ & 1.125 & 0.006 & 0.057 & 0.005 & 0.2834 & 0.0903 \\
\hline E2 & + & $0.3 \pm 0 \%$ & $25.6 \pm 1.9 \%$ & $0.4 \pm 0.1 \%$ & $28.1 \pm 1.6 \%$ & 1.476 & 0.002 & 0.033 & 0.004 & 0.2590 & 0.1004 \\
\hline E3 & + & $3.6 \pm 0.8 \%$ & $7.4 \pm 0.9 \%$ & $0.5 \pm 0.2 \%$ & $6.5 \pm 1 \%$ & 0.606 & 0.003 & 0.030 & 0.008 & 0.1925 & 0.0907 \\
\hline E4 & + & $0.3 \pm 0 \%$ & $36.3 \pm 12.2 \%$ & $0.4 \pm 0.3 \%$ & $33.9 \pm 11.7 \%$ & 0.734 & 0.003 & 0.036 & 0.006 & 0.1096 & 0.0288 \\
\hline E5 & & $16 \pm 18.9 \%$ & $85 \pm 10.3 \%$ & $50.5 \pm 14.8 \%$ & $71.6 \pm 8.1 \%$ & 0.624 & 0.001 & 0.018 & 0.003 & 0.0920 & 0.0404 \\
\hline E6 & & $0.2 \pm 0.1 \%$ & $42.5 \pm 6.1 \%$ & $3.3 \pm 1.4 \%$ & $26.6 \pm 4.6 \%$ & 0.783 & 0.001 & 0.032 & 0.006 & 0.0764 & 0.0328 \\
\hline E7 & & $110.9 \pm 4.9 \%$ & $103.5 \pm 4.4 \%$ & $108.1 \pm 2.3 \%$ & $92.5 \pm 10.1 \%$ & - & - & - & - & 0.2565 & 0.0257 \\
\hline S1 & + & $0.1 \pm 0 \%$ & $5.1 \pm 0.2 \%$ & $0 \pm 0 \%$ & $65.3 \pm 3.7 \%$ & 3.037 & 0.007 & 0.214 & 0.008 & 0.1084 & 0.1511 \\
\hline S2 & + & $0.1 \pm 0 \%$ & $0 \pm 0 \%$ & $0 \pm 0 \%$ & $0 \pm 0 \%$ & 2.111 & 0.015 & 0.148 & 0.030 & 0.0743 & 0.0266 \\
\hline S3 & + & $85.2 \pm 4.9 \%$ & $75 \pm 1.9 \%$ & $74.8 \pm 0.6 \%$ & $83.9 \pm 1 \%$ & - & - & - & - & 0.2639 & 0.0700 \\
\hline S4 & & $58.7 \pm 5.7 \%$ & $72 \pm 5 \%$ & $33.4 \pm 5.7 \%$ & $47.3 \pm 4.7 \%$ & - & - & - & - & 0.0338 & 0.0246 \\
\hline S5 & & $87.5 \pm 18.2 \%$ & $101.4 \pm 4.6 \%$ & $106.7 \pm 3.8 \%$ & $98.7 \pm 2 \%$ & - & - & - & - & 0.1251 & 0.0176 \\
\hline S6 & & $67.3 \pm 12.1 \%$ & $61.8 \pm 9.4 \%$ & $67.4 \pm 14.4 \%$ & $63.2 \pm 10.4 \%$ & - & - & - & - & 0.0277 & 0.0187 \\
\hline S7 & & $105.2 \pm 4.9 \%$ & $90.3 \pm 3.4 \%$ & $95.7 \pm 3.6 \%$ & $89.8 \pm 2.9 \%$ & - & - & - & - & 0.0930 & 0.0738 \\
\hline S8 & & $100.8 \pm 2.5 \%$ & $88 \pm 4.8 \%$ & $87.9 \pm 4 \%$ & $92.2 \pm 3.5 \%$ & - & - & - & - & 0.0867 & 0.0149 \\
\hline 59 & & $0.3 \pm 0.2 \%$ & $0.1 \pm 0.2 \%$ & $0.2 \pm 0.1 \%$ & $0.4 \pm 0.1 \%$ & 2.982 & 0.012 & 0.206 & 0.018 & 0.0221 & 0.0553 \\
\hline 510 & & $0.1 \pm 0.1 \%$ & $0.1 \pm 0.1 \%$ & $0.1 \pm 0 \%$ & $0.2 \pm 0 \%$ & 2.669 & 0.021 & 0.178 & 0.032 & 0.1954 & 0.1393 \\
\hline
\end{tabular}

${ }^{\text {a }}$ Species marked by ' + ' accepted sinigrin as the sole carbon source

${ }^{b}$ Data for other glucosinolates is available in Additional file 3: Table 2

Decomposing ability was tested in pure horseradish extract as the growth medium. $I C_{50}$ values of the isothiocyanates of horseradish against growth of fungi are given in end-concentration in the medium (mM). Fungi: E1, Fusarium oxysporum; E2, Macrophomina phaseolina; E3, Fusarium oxysporum; E4, Setophoma terrestris; E5, Paraphoma radicina; E6, Paraphoma radicina; E7, Oidiodendron cerealis; S1-S10: soil fungi from the same site as one of the horseradish samples

Abbreviations: AITC allyl isothiocyanate, GBR glucobrassicin, GIB glucoiberin, GLN gluconasturtiin, n.d. not determined, PEITC 2-phenylethyl-isothiocyanate, SGN sinigrin

a

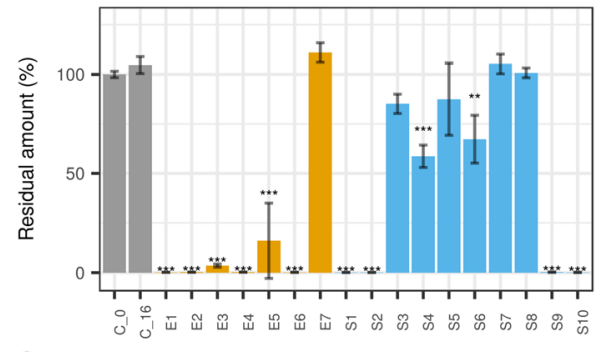

C

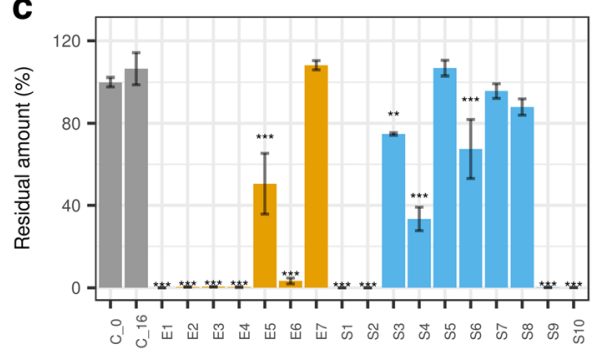

b

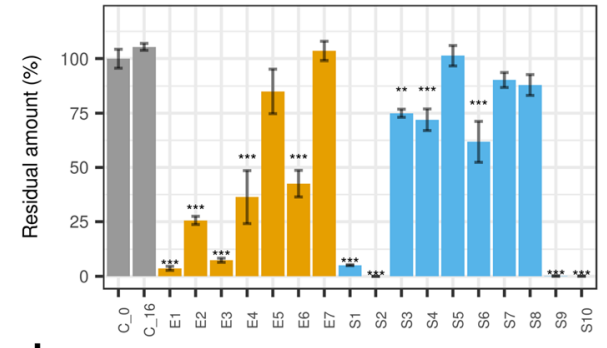

d

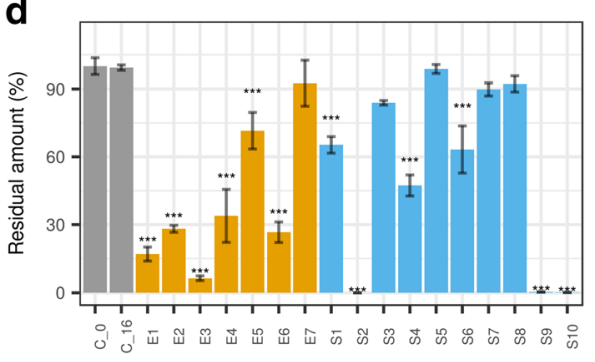

Fig. 3 Decomposition of glucosinolates in horseradish extract incubated with endophytic and soil fungi for 16 days. Data are given for a single vial (equivalent to $0.295 \mathrm{~g}$ FW horseradish root). Subplots: a., sinigrin; b., glucoiberin; c., gluconasturtiin; d., glucobrassicin. Fungi: E1, Fusarium oxysporum; E2, Macrophomina phaseolina; E3, Fusarium oxysporum; E4, Setophoma terrestris; E5, Paraphoma radicina; E6, Paraphoma radicina; E7, Oidiodendron cerealis; C_O, control (zero time); C_16, control (end-time); S1-S10: soil fungi from the same site as one of the horseradish samples. Statistical test: Dunnett's test, end-time samples were compared to end-time control $\left(n=3,{ }^{* *}, p<3^{*} 10^{-6} ; *^{*}, p<3^{*} 10^{-5}, p<1.9^{*} 10^{-4}\right)$. For the data in Table form, see Additional file 2 Table 52 


\section{Detection of decomposition products}

We succesfully detected allyl isothiocyanate during decomposition of sinigrin by endophytes growing on horseradish extract solidified with agar. An SPME-GCMS approach was necessary. Also, the glutathione conjugate of the allyl isothiocyanate was detected from the media by LC-MS.

The LC-MS data from the HRE incubation were first screened for desulfated glucosinolates using a modified protocol of [49]. The sulfatase enzyme from Helix pomatia worked well on both pure glucosinolates and the HRE. The desulfo-GSLs gave characteristic peaks in positive ion mode LC-MS (not shown), but none of these products were present in the HRE incubated with the endophytic fungi.

We also attempted to detect typical volatile decomposition products of sinigrin or gluconasturtiin, namely ITCs or nitriles. In the absence of specifier proteins or other special conditions, the decomposition products of glucosinolates are the isothiocyanates (Fig. 1) [13], which are highly toxic fungicides $[15,26]$. Therefore the detection of isothiocyanates and the decomposition of glucosinolates infers myrosinase (thioglucosidase) activity in the fungi. Endophyte inoculation significantly influenced AITC concentration in the headspace as shown by GC-MS (Additional file 1: Figure S6a; $p<0.01$ ). Surprisingly, only $E 5$ and $E 6$ caused a detectable release of AITC into the air of the Petri dish (Fig. 4, Additional file 1: Figure S6a). About $1.25 \%$ of the total amount of ITC was released into the air

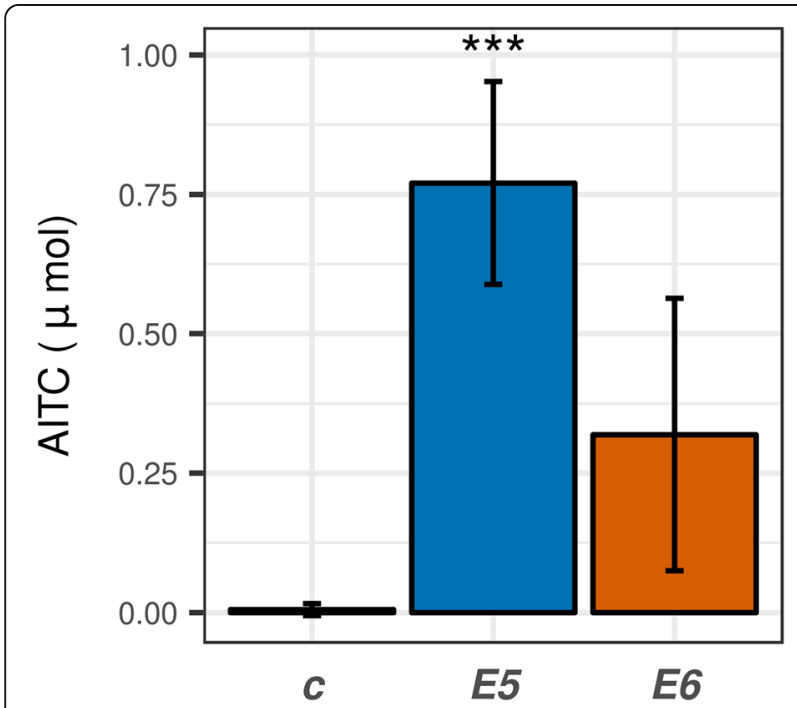

Fig. 4 Allyl isothiocyanate content above the endophytic fungi growing on solidified horseradish extract. SPME was carried out on the 6th day for $24 \mathrm{~h}$. The data were obtained by SPME-GC-MS, on day $6(n=3)$. Fungi: E5, Paraphoma radicina; E6, Paraphoma radicina; $C$, control (not inoculated). Statistical test: Dunnett's test, 6th day samples compared to 6th day control $\left(n=3,{ }^{* * *}, p<10^{-3} ;{ }^{* *}, p<\right.$ $\left.10^{-2 *}, p<0.05\right)$ within $24 \mathrm{~h}$ on day 6 (E6). The other four SGN decomposing species (E1-E4) emitted trace amounts of AITC only. The nitrile generation would be a way to avoid toxicity of ITCs, and is shown in Aspergillus [17] where the product is nitrile. Despite this fact, allyl cyanide was not detected from any of the samples (Additional file 1: Figure S6b). No significant decomposition products of gluconasturtiin could be detected either (Additional file 1: Figure S6c), which could also be the result of their low volatility compared to AITC and allyl nitrile. The extracellular production of ITCs by E5-6 can give these strains a competitive advantage over more sensitive species, as the released amount can result in concentrations inhibitory to fungi. However, ITCs are only detectable by GC-MS in their free form, the conjugates are not volatile. For example, despite that the liquid medium was directly extracted with organic solvent and analyzed by GC-MS, volatile products could not be detected during the rapid decomposition of sinigrin by Citrobacter [19]. It is possible that AITC was present in the medium only in conjugate form.

As the volatile breakdown products were not found in case of many strains, a targeted search was run to find possible non-volatile GSL metabolites (CysGly - AITC, Cys - AITC, glutathione - AITC) from the LC-MS profiles was run. This approach revealed a peak with $\mathrm{m} / \mathrm{z}$ 405.0914 at a retention time of $8.12 \mathrm{~min}$. The compound was suspected to be GSH (glutathione) - AITC conjugate based on its exact mass. Its structure was confirmed by synthesizing the identical compound by mixing pure GSH and AITC in buffer, using the derivatization reaction employed in our recent study [33]. The two compounds showed identical $\mathrm{MS}^{2}$ spectra and retention times (Additional file 1: Figure S7). The GSH - AITC adduct concentration reached its maximum during the high-rate sinigrin decomposition for most strains (Figs. $2 a, 5)$. The media of those fungi that did not release the AITC in the environment also contained high amounts of the GSH - AITC adduct.

Compared to zero-time control, the media of $E 1$ and $E 2$ contained 8.77 and 6.07-fold more GSH - AITC, respectively. This difference was much more expressed in the media of E4, E5 and E6 (29.14, 14.05 and 43.27-fold increase, respectively, $p<0.01$, Dunnett's test). E3 did not cause any increase, which is likely to be the result of the flat decomposition curve for sinigrin (Figs. 2a, 5, Table 3). The presence of GSH - AITC adduct suggests that the ITCs are at least in part the decomposition products of GSLs in all fungi. AITC spontaneously conjugates with the thiol pool of the fungi (mainly GSH [50]) and is in part released into the medium. ITC toxicity increases when the GSH pool of the fungi becomes depleted [16], resulting in increased attack on the protein -SH groups. The rapid recovery of GSH from the conjugate could result in such a low detected amounts of GSH-AITC (Fig. 5). Recovery 


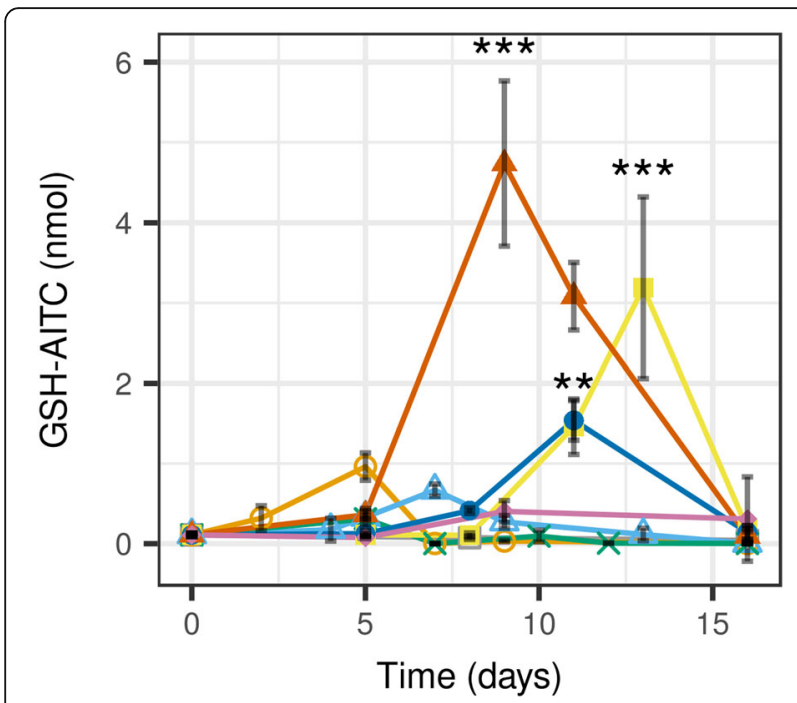

Fig. 5 Change of concentration of a sinigrin metabolite, allyl isothiocyanate - glutathione adduct in horseradish extract inoculated by endophytic fungi from horseradish. Fungi: E1, Fusarium oxysporum; E2, Macrophomina phaseolina; E3, Fusarium oxysporum; E4, Setophoma terrestris; E5, Paraphoma radicina; E6, Paraphoma radicina; E7, Oidiodendron cerealis; $C$, control (not inoculated). Statistical test: Dunnett's test, time points of highest concentrations (different for each strain) were compared to zero-time controls ( $n=$ $\left.3,{ }^{* * *}, p<10^{-3} ; * *, p<10^{-2 *}, p<0.05\right)$

can be spontaneous or it can be catalyzed by glutathione-S transferase enzymes $[51,52]$. The tolerance to AITC in the necrotrophic fungus Alternaria brassicicola was also characterized by elevation of glutathione-S transferases, and thioredoxins [27] The GSH-AITC can either be present in the medium as a result of active efflux or lysis of dead cells.

Even when GSH-AITC was detected, free GSH could also be detected from the medium in higher amounts. This suggest that the GSH pool was not depleted under the current conditions, and/or an active recovery mechanism is operating at high efficacy. The presence of free GSH also explains the observed viability of fungi during sinigrin decomposition. It is also possible that GSH is actively secreted into the medium, though only very limited extracellular functions of the molecule are described in fungi [50]. The high intracellular concentration and rapid recycling of GSH would also explain the findings of Albaser et al. [19]. They have shown that while the purified Citrobacter myrosinase decomposed pure glucosinolates into ITCs, AITC could not be detected from the medium of Citrobacter growing on sinigrin as the sole carbon source [19]. It is likely that the endophytic fungi of the current study behaved similarly to the mentioned Citrobacter strain during glucosinolate decomposition. Of course, production of a different, non-detected product or conjugate cannot be ruled out either.
Altogether, enzymes having thioglucosidase activity can be expected to be behind the observed GSL decomposition, as shown in a few instances [17, 18, 47]. The product was ITC, found either in free or conjugate form. Like the enzyme of Citrobacter examined by [19], these enzymes can also be adapted glucosidases (or homologues) that also accept thioglucosides as substrates. Figures 2 and 3 show that these microbial enzymes likely have wide specificity: they accept most glucosinolates as substrates, but the different GSLs are decomposed with different velocity. Different decomposition speeds against GSLs with different side-chains have also been described for plant myrosinases $[33,53]$ and the myrosinase of Citrobacter [19]. Despite that fungi seem to tolerate the released ITC quite well during growth on HRE, questions still remain. Decomposition does not necessarily mean the ability to selectively utilize the plant compounds. Therefore, growth on sinigrin as the sole carbon source was tested.

\section{The glucosinolate sinigrin as the sole carbon source for endophytic and soil fungi}

Testing of the growth of fungi in media with sinigrin as the sole carbon source revealed that many strains have the ability to utilize the plant defensive compounds. In this case, a more marked difference between soil fungi and endophytes was found.

Surprisingly, four of seven horseradish endophytes (E1-4) accepted sinigrin as the sole carbon source $(p<$ $0.05)$. The most efficient sinigrin decomposer performed well on both sinigrin and glucose as carbon sources $(E 1$, Figs. 2a, 6). Less efficient decomposers showed growth but apparently sinigrin provided less favorable conditions than glucose (E2-4, Fig. 6). This is another good example of within-species variability ( $E 1$ vs $E 3)$.

When subjecting the soil fungi to the same experiment, only three of ten strains were capable of using sinigrin as the sole carbon source $(p<0.05$, Fig. 6). Therefore this phenomenon seems to be more specific than GSL decomposition. Though the endophytes could not be clearly separated from the soil fungi by their GSL decomposition ability, the chance to find endophytes accepting sinigrin as the sole carbon source was 1.7-fold higher compared to that of the soil fungi. One strain could not even develop on the Czapek Dox medium (S7), perhaps due to the lack of a favorable organic $\mathrm{N}$ source. An active strain (S1) was found to belong to the same species as E1, E3 based on morphology and its ITS sequence. One could think that Fusarium sp. tend to have some enzyme that decomposes GSLs, which may be an explanation behind the high prevalence of Fusarium strains in the root microbiome of Brassicaceae species [25]. However, S3, S7 were also identified as Fusarium sp. based on ITS and eF1 sequences as well as their morphological characteristics. These strains 


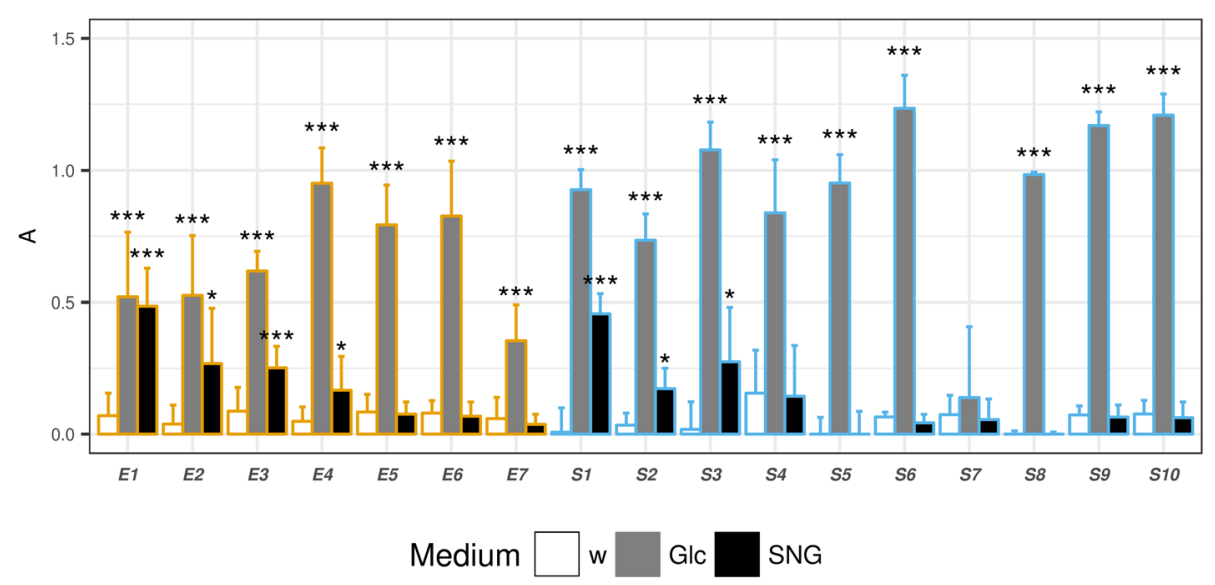

Fig. 6 Growth of horseradish endophytes and soil fungi in Czapek Dox liquid media with different carbon sources. Data were obtained by measuring increased light absorbance (A) at $800 \mathrm{~nm}$ by a plate reader. Carbon sources: Glc, 2\% glucose; SGN, sinigrin (equimolar to $2 \%$ glucose). Control is pure water (W). Fungi: Fungi: E1, Fusarium oxysporum; E2, Macrophomina phaseolina; E3, Fusarium oxysporum; E4, Setophoma terrestris; E5, Paraphoma radicina; E6, Paraphoma radicina; E7, Oidiodendron cerealis; S1-S10: soil fungi from the same site as one of the horseradish samples. Statistical test: Dunnett's test, end-time samples were compared to end-time controls, for each fungus separately $\left(n=3,{ }^{* * *}, p<10^{-3} ;{ }^{* *}, p<10^{-2}\right.$ $*, p<0.05)$

showed much less glucosinolate decomposing potential (Fig. 3, Table 3, Additional file 1: Figure S5). The above highlight the significance of within-species variability in similar approaches. Therefore, more studies are required to fully explain the potential interaction between the GSLs and Fusarium sp. Other sinigrin-utilizing microbes have been described before, e.g. some strains of Aspergillus sp. $[17,54]$ and bacteria $[19,23,55]$. It is worth mentioning that this activity can be achieved by expressing a widespecificity $\beta$-O-glucosidase as in the case of Citrobacter [19]. This might have been the case in the current study.

Evidently, we can state that the endophytes from horseradish roots are rich sources of enzymes that can break down the secondary metabolites of the host plant. Moreover, these strains are able to use the defensive metabolites as nutrients to develop, which ability is less abundant among the soil fungi. This is interesting in the light of the fact that these defensive metabolites give rise to antifungal compounds upon decomposition. The enzymes responsible for the breakdown of the glucosinolates can be specific thioglucosidases, or $\beta$-O-glucosidases with wider specificity as well. The above results suggest the adaptation of the endophytes to the defensive metabolites of the plant, which is highlighted by the higher chance to find active endophytes than soil fungi. After investigating the effects of the endophytes on the plant metabolites, the effects of the ITCs on the fungi were also examined, with special regard to the soil fungi - endophyte difference.

\section{Growth inhibition by isothiocyanates}

Relatively low amounts of ITCs successfully inhibited the growth of all tested fungi in liquid media. The inhibition data with the fitted curves and $\mathrm{IC}_{50}$ calculation are shown in Additional file 1: Figures. S8 and S9. Again, a difference was found between the endophyte and the soil fungal group: the endophytes tolerated AITC better.

It is apparent from the $\mathrm{IC}_{50}$ values in Table 3 that most fungal strains were more tolerant to AITC than PEITC: the average difference between the $\mathrm{IC}_{50}$ values is 2.30 -fold. The difference is likely to be a consequence of the different stability of the ITC adducts with proteins [51], as well as the different polarity and therefore different solubility in water, different kinetics of the ITCs themselves.

If we recall the AITC release data from the SPME-GCMS experiment (Fig. 4), we get a theoretical $0.07 \mathrm{mM}$ concentration for AITC in the liquid medium, assuming complete dissolution in the medium which is usually the case [56]. By comparing this with the $\mathrm{IC}_{50}$ data (Table 3 ), it is apparent that the AITC is released from horseradish extract by $E 5$ and $E 6$ is enough to theoretically inhibit growth of vulnerable strains $(S 2, S 6, S 9)$. The same amount would also slow the growth of $E 5$ and $E 6$ as well, perhaps that is an another reason why the GSL decomposing enzymes are not expressed until the end of growth.

By carefully comparing the distribution of the $\mathrm{IC}_{50}$ values of the two groups (endophytes vs soil fungi, Table 3), it can be observed that the endophytic group almost lacks fungi with high vulnerablity to AITC. Only two of seven endophytes had an $\mathrm{IC}_{50}$ below $0.1 \mathrm{mM}$, while more than half of the soil fungi fell in the same range. By directly comparing the median $\mathrm{IC}_{50}$ values, a two-fold advantage of the endophytes was recognized: the median $\mathrm{IC}_{50}$ values for soil fungi and endophytes were 0.090 and $0.192 \mathrm{mM}$ AITC, respectively. This difference is not found in the case of PEITC: median $\mathrm{IC}_{50}$ values for soil fungi and endophytes were 0.040 and 0 . 
$041 \mathrm{mM}$, respectively. As the phenomenon is extremely overlaid by interspecies variability, further studies will be necessary to provide further evidence on this logically expectable adaptation to ITCs. The background mechanisms behind the adaptation likely include genes that enable enhanced recycling or excessive production of thiols [27], such as GSH [16, 50]. This can lead to resistance to fungicides that target the thiol pool of phytopathogenic fungi $[57,58]$.

The difference of the sensitivity of the fungi to the two tested ITCs is a fine example of the mechanism behind the plant's ability to drive the development of its microbiome [59]. It also exemplifies the rationale behind producing various ITC precursors for chemical defense. Actually, the mixture of ITCs can limit activity of much more strains than AITC or PEITC alone would be capable of, without even taking synergistic effects into account [16].

\section{Conclusions}

Our main conclusion is that endophytes are active participants of the glucosinolate - myrosinase - isothiocyanate system. The present work also provides preliminary data that suggest existence of a detectable adaptation of the endophytes to the host plant's antifungal secondary metabolites. This adaptation distinguished the endophytic fungi from the soil fungi from the same soil to some extent. We used various experimental approaches to find different manifestations of this adaptation. These were the following: 1 ., The endophytes were more tolerant to the plant defensive compound allyl isothiocyanate than the soil fungi. 2., Compared to soil fungi, a higher proportion of endophytic strains were capable of glucosinolate decomposition. 3., Compared to soil fungi, a higher proportion of endophytes could utilize sinigrin as the sole carbon source. The results have shown that no clear boundary can be drawn between the root endophytes and soil fungi, but still, there is a detectable difference. The case is similar to that found when the ratio of new natural products from endophytes and soil fungi was compared [60]. The results also fit the endophytic continuum approach very well: endophytes can be present at many points along a functional gradient. The phenomena found at the same time show examples of mechanisms by which plants drive the development of their root microbiome by exudates. The root endophytes found are likely a subset of the available soil fungi that have the biochemical potential and opportunity to colonize the plant's roots. Their competitive advantage came from the higher potential tolerance of the plant's defensive compounds, among others. If the fungal myrosinase is expressed in planta while the plant is still healthy, this may be a possible mechanism by which endophytes decrease incidence of plant diseases in Brassicaceae species. The same would result in changes in the plant microbiome that would be controlled by endophytes by releasing plant defensive metabolites. It can also be a means by which the endophytes override plant chemical defense reactions. Using a myrosinase is a way to disarm the chemical bomb of glucosinolates that the fungus will encounter upon infection of the plant root. As many other interesting possibilities exist, we think that the myrosinase activity and the chemical adaptation of endophytes warrants further studies.

\section{Additional files}

Additional file 1: Figures S1-S9. Supplementary Figures (including Compound structures; Decomposition of minor glucosinolates in horseradish extract by endophytic and soil fungi; Sinigrin decomposition by endophytes in sinigrin-supplemented Saboraud Glucose Broth; Growth curves; SPME-GC-MS chromatograms; LC-MS extracted ion chromatograms; Growth inhibition curves and $\mathrm{IC}_{50}$ calculation) and additional methodical details on SPME-GC-MS. (PDF $5696 \mathrm{~kb}$ )

Additional file 2: Table S1. Changes in concentration of glucosinolates in horseradish extracts incubated with horseradish endophytes. Raw abundance data. (DOC $58 \mathrm{~kb}$ )

Additional file 3: Table S2. Residual amount of sinigrin and minor glucosinolates in horseradish extract after 16 days of incubation with endophytic fungi from horseradish and soil fungi. (DOC $33 \mathrm{~kb}$ )

\section{Abbreviations}

AITC: allyl isothiocyanate; CzD: Czapek Dox medium; GC-MS: Gas Chromatography - Mass Spectrometry; GSH: Glutathione, reduced form; GSL : Glucosinolate; HRE: horseradish extract; ITC: Isothiocyanate; LC-MS : High Performance Liquid Chromatography - Mass Spectrometry; MYR: myrosinase; PDA: Potato dextrose agar; PEITC: 2-phenylethyl isothiocyanate;

PES: Polyethersulfone membrane; SGA: Saboraud Glucose Agar; SPME: Solid phase micro extraction

\section{Acknowledgements}

We thank our reviewers for the suggestions and comments.

\section{Funding}

The study was supported by the Hungarian Scientific Research Fund (OTKA) research grants No. 112374 and 124339. This work was partially supported by the European Union and the European Social Fund through project EFOP3.6.1-16-2016-00022.

\section{Availability of data and materials}

The datasets used and/or analysed during the current study are available from the corresponding author on reasonable request.

\section{Authors' contributions}

Study conception and design: S G, acquisition of data: Zs Sz (all methods), T $P$ (growth inhibition, carbon source experiments), Z C (LC-MS), A K Sz (LCMS), T E (identification of fungi), R B (SPME-GC-MS), L T S (LC-MS); interpretation of data: S G, Zs Sz; drafting of manuscript: S G, Critical revision: $\mathrm{G}$ V. All authors read and approved the final manuscript.

\section{Competing interests}

The authors declare that they have no competing interests.

\section{Publisher's Note}

Springer Nature remains neutral with regard to jurisdictional claims in published maps and institutional affiliations.

\section{Author details}

'Department of Botany, Division of Pharmacognosy, University of Debrecen, Egyetem tér 1, Debrecen H-4010, Hungary. ${ }^{2}$ Department of Organic Chemistry, University of Debrecen, Egyetem tér 1, Debrecen H-4010, Hungary. ${ }^{3}$ Department of Biotechnology and Microbiology, University of 
Debrecen, Egyetem tér 1, Debrecen $\mathrm{H}-4010$, Hungary. ${ }^{4}$ Department of Pharmacognosy, Semmelweis University, Üllői út 26, Budapest H-1085, Hungary. ${ }^{5}$ Agricultural and Molecular Research and Service Institute, University of Nyíregyháza, Sóstói str. 31/b, Nyíregyháza H-4400, Hungary.

\section{Received: 28 April 2017 Accepted: 26 April 2018 Published online: 09 May 2018}

\section{References}

1. van der Heijden MGA, Hartmann M. Networking in the Plant Microbiome. PLoS Biol. 2016;14:e1002378.

2. Ludwig-Müller J. Plants and endophytes: equal partners in secondary metabolite production? Biotechnol Lett. 2015;37:1325-34.

3. Schulz B, Boyle C. The endophytic continuum. Mycol Res. 2005;109:661-86.

4. Fesel PH, Zuccaro A. Dissecting endophytic lifestyle along the parasitism/ mutualism continuum in Arabidopsis. Curr Opin Microbiol. 2016;32:103-12.

5. Gunatilaka AAL. Natural Products from Plant-Associated Microorganisms: Distribution, Structural Diversity, Bioactivity, and Implications of Their Occurrence $\perp$. J Nat Prod. 2006;69:509-26.

6. Suryanarayanan TS, Thirunavukkarasu N, Govindarajulu MB, Gopalan V. Fungal endophytes: An untapped source of biocatalysts. Fungal Divers. 2012;54:19-30

7. Vos CM, Yang Y, De Coninck B, Cammue BPA. Fungal (-like) biocontrol organisms in tomato disease control. Biol Control. 2014;74:65-81.

8. Rajulu MBG, Thirunavukkarasu N, Suryanarayanan TS, Ravishankar JP, Gueddari NEE, Moerschbacher BM. Chitinolytic enzymes from endophytic fungi. Fungal Divers. 2011;47:43-53.

9. Slippers B, Wingfield MJ. Botryosphaeriaceae as endophytes and latent pathogens of woody plants: diversity, ecology and impact. Fungal Biol Rev. 2007:21:90-106.

10. Photita W, Lumyong S, Lumyong P, McKenzie EHC, Hyde KD. Are some endophytes of Musa acuminata latent pathogens? Fungal Divers. 2004;16:131-40.

11. Kissen R, Rossiter JT, Bones AM. The 'mustard oil bomb': not so easy to assemble?! Localization, expression and distribution of the components of the myrosinase enzyme system. Phytochem Rev. 2009;8:69-86.

12. Nguyen NM, Gonda S, Vasas G. A Review on the Phytochemical Composition and Potential Medicinal Uses of Horseradish (Armoracia rusticana) Root. Food Rev Int. 2013;29:1-15.

13. Wittstock U, Burow M. Tipping the Scales-Specifier Proteins in Glucosinolate Hydrolysis. IUBMB Life. 2007:59:744-51.

14. Wu H, Zhang $X$, Zhang G-A, Zeng S-Y, Lin K-C. Antifungal Vapour-phase Activity of a Combination of Allyl Isothiocyanate and Ethyl Isothiocyanate Against Botrytis cinerea and Penicillium expansum Infection on Apples. J Phytopathol. 2011:159:450-5.

15. Azaiez I, Meca G, Manyes L, Fernández-Franzón M. Antifungal activity of gaseous allyl, benzyl and phenyl isothiocyanate in vitro and their use for fumonisins reduction in bread. Food Control. 2013;32:428-34.

16. Bertóti R, Vasas G, Gonda S, Nguyen NM. Sz\Hoke É, Jakab Á, et al. Glutathione protects Candida albicans against horseradish volatile oil. J. Basic Microbiol. 2016;56:1071-9.

17. Galletti S, Sala E, Leoni O, Cinti S, Cerato C. Aspergillus flavus transformation of glucosinolates to nitriles by an arylsulfatase and a $\beta$-thio-glucosidase. Soil Biol Biochem. 2008:40:2170-3.

18. Smits JP, Knol W, Bol J. Glucosinolate degradation by Aspergillus clavatus and Fusarium oxysporum in liquid and solid-state fermentation. Appl Microbiol Biotechnol. 1993;38:696-701.

19. Albaser A, Kazana E, Bennett MH, Cebeci F, Luang-In V, Spanu PD, et al. Discovery of a Bacterial Glycoside Hydrolase Family 3 (GH3) $\beta$-Glucosidase with Myrosinase Activity from a Citrobacter Strain Isolated from Soil. J Agric Food Chem. 2016;64:1520-7.

20. Tani N, Ohtsuru M, Hata T. Purification and general characteristics of bacterial myrosinase produced by Enterobacter cloacae. Agric Biol Chem. 1974;38:1623-30.

21. Saha S, Hollands W, Teucher B, Needs PW, Narbad A, Ortori CA, et al. Isothiocyanate concentrations and interconversion of sulforaphane to erucin in human subjects after consumption of commercial frozen broccoli compared to fresh broccoli. Mol Nutr Food Res. 2012;56:1906-16.

22. Krul C, Humblot C, Philippe C, Vermeulen M, van Nuenen M, Havenaar R, et al. Metabolism of sinigrin (2-propenyl glucosinolate) by the human colonic microflora in a dynamic in vitro large-intestinal model. Carcinogenesis. 2002; 23:1009-16.
23. Luang-In V, Narbad A, Nueno-Palop C, Mithen R, Bennett M, Rossiter JT. The metabolism of methylsulfinylalkyl- and methylthioalkyl-glucosinolates by a selection of human gut bacteria. Mol Nutr Food Res. 2014;58:875-83.

24. Tian Y, Amand S, Buisson D, Kunz C, Hachette F, Dupont J, et al. The fungal leaf endophyte Paraconiothyrium variabile specifically metabolizes the hostplant metabolome for its own benefit. Phytochemistry. 2014;108:95-101.

25. Ishimoto H, Fukushi Y, Yoshida T, Tahara S. Rhizopus and Fusarium are selected as dominant fungal genera in rhizospheres of Brassicaceae. J Chem Ecol. 2000;26:2387-99

26. Wu X-M, Meijer J. In vitro degradation of intact glucosinolates by phytopathogenic fungi of Brassica. Proc. 10th Int. Rapeseed Congr. [Internet]. 1999 [cited 2017 Jan 30]. p. 26-29. Available from: http://www. regional.org.au/au/gcirc/3/617.htm

27. Sellam A, Dongo A, Guillemette T, Hudhomme P, Simoneau P. Transcriptional responses to exposure to the brassicaceous defence metabolites camalexin and allyl-isothiocyanate in the necrotrophic fungus Alternaria brassicicola. Mol Plant Pathol. 2007;8:195-208.

28. Hallmann J, Berg G, Schulz B. Isolation Procedures for Endophytic Microorganisms. In: Schulz PDBJE, Boyle DCJC, Sieber DTN, editors. Microb. Root Endophytes [Internet]. Springer Berlin Heidelberg; 2006 [cited 2014 Aug 22]. p. 299-319. Available from: http://link.springer.com/chapter/10. 1007/3-540-33526-9_17

29. Gonda S, Kiss A, Emri T, Batta G, Vasas G. Filamentous fungi from Plantago lanceolata L. leaves: Contribution to the pattern and stability of bioactive metabolites. Phytochemistry. 2013;86:127-36.

30. Gowda H, Ivanisevic J, Johnson CH, Kurczy ME, Benton HP, Rinehart D, et al. Interactive XCMS Online: Simplifying Advanced Metabolomic Data Processing and Subsequent Statistical Analyses. Anal Chem. 2014:86:6931-9.

31. R Core Team. R: A language and environment for statistical computing [Internet]. Vienna, Austria; 2014. Available from: http://www.R-project.org

32. Wickham H. ggplot2: Elegant Graphics for Data Analysis. Verlag New York: Springer; 2009.

33. Gonda S, Kiss-Szikszai A, Szűcs Z, Nguyen NM, Vasas G. Myrosinase Compatible Simultaneous Determination of Glucosinolates and Allyl Isothiocyanate by Capillary Electrophoresis Micellar Electrokinetic Chromatography (CE-MEKC). Phytochem Anal. 2016;27:191-8.

34. Ritz C, Baty F, Streibig JC, Gerhard D. Dose-Response Analysis Using R. PLoS One. 2015;10:e0146021.

35. Zhang Q, Zhang J, Yang L, Zhang L, Jiang D, Chen W, et al. Diversity and biocontrol potential of endophytic fungi in Brassica napus. Biol Control. 2014:72:98-108

36. de Gruyter J, Aveskamp MM, Woudenberg JHC, Verkley GJM, Groenewald JZ, Crous PW. Molecular phylogeny of Phoma and allied anamorph genera: Towards a reclassification of the Phoma complex. Mycol Res. 2009;1 13:508-19.

37. Chowdhary K, Kaushik N. Fungal Endophyte Diversity and Bioactivity in the Indian Medicinal Plant Ocimum sanctum Linn. PLoS One. 2015;10:e0141444.

38. El-Elimat T, Figueroa M, Raja HA, Graf TN, Swanson SM, Falkinham JO, et al. Biosynthetically Distinct Cytotoxic Polyketides from Setophoma terrestris. Eur. J. Org. Chem. 2015;2015:109-121.

39. MCLEAN CB, Cunnington JH, Lawrie AC. Molecular diversity within and between ericoid endophytes from the Ericaceae and Epacridaceae. New Phytol. 1999;144:351-8.

40. Grelet G, Martino E, Dickie IA, Tajuddin R, Artz R. Ecology of ericoid mycorrhizal fungi: what insight have we gained with molecular tools and what's missing. Mol Mycorrhizal Symbiosis Springer. 2016:405-19.

41. Rochfort SJ, Trenerry VC, Imsic M, Panozzo J, Jones R. Class targeted metabolomics: ESI ion trap screening methods for glucosinolates based on MSn fragmentation. Phytochemistry. 2008;69:1671-9.

42. Fabre N, Poinsot V, Debrauwer L, Vigor C, Tulliez J, Fourasté I, et al. Characterisation of glucosinolates using electrospray ion trap and electrospray quadrupole time-of-flight mass spectrometry. Phytochem Anal. 2007;18:306-19.

43. Agneta R, Lelario F, De Maria S, Möllers C, Bufo SA, Rivelli AR. Glucosinolate profile and distribution among plant tissues and phenological stages of field-grown horseradish. Phytochemistry. 2014;106:178-87.

44. Tian Q, Rosselot RA, Schwartz SJ. Quantitative determination of intact glucosinolates in broccoli, broccoli sprouts, Brussels sprouts, and cauliflower by high-performance liquid chromatography-electrospray ionization-tandem mass spectrometry. Anal Biochem. 2005;343:93-9.

45. Maldini M, Baima S, Morelli G, Scaccini C, Natella F. A liquid chromatography-mass spectrometry approach to study "glucosinoloma" in broccoli sprouts. J Mass Spectrom. 2012;47:1198-206. 
46. Murphy BR, Batke SP, Doohan FM, Hodkinson TR. Media Manipulations and the Culture of Beneficial Fungal Root Endophytes. Int J Biol. 2015;7:94.

47. Reese ET, Clapp RC, Mandels M. A thioglucosidase in fungi. Arch Biochem Biophys. 1958;75:228-42.

48. Robinson KO, Beyene DA, van Berkum P, Knight-Mason R, Bhardwaj HL. Variability in plant-microbe interaction between Lupinus lines and Bradyrhizobium strains. Plant Sci. 2000;159:257-64.

49. Ratzka A, Vogel H, Kliebenstein DJ, Mitchell-Olds T, Kroymann J. Disarming the mustard oil bomb. Proc Natl Acad Sci. 2002;99:11223-8.

50. Pócsi I, Prade RA, Penninckx MJ. Glutathione, altruistic metabolite in fungi. Adv Microb Physiol. 2004:49:1-76.

51. Conaway CC, Krzeminski J, Amin S, Chung FL. Decomposition rates of isothiocyanate conjugates determine their activity as inhibitors of cytochrome P450 enzymes. Chem Res Toxicol. 2001;14:1170-6.

52. Zhang YS, Kolm RH, Mannervik B, Talalay P. Reversible Conjugation of Isothiocyanates with Glutathione Catalyzed by Human Glutathione Transferases. Biochem Biophys Res Commun. 1995;206:748-55.

53. Bernardi R, Finiguerra MG, Rossi AA, Palmieri S. Isolation and Biochemical Characterization of a Basic Myrosinase from Ripe Crambe abyssinica Seeds, Highly Specific for epi-Progoitrin. J Agric Food Chem. 2003;51:2737-44.

54. Wang $X$, Jin $Q$, Wang T, Huang J, Xia Y, Yao L, et al. Screening of glucosinolate-degrading strains and its application in improving the quality of rapeseed meal. Ann Microbiol. 2012;62:1013-20.

55. Brabban $A D$, Edwards C. Isolation of glucosinolate degrading microorganisms and their potential for reducing the glucosinolate content of rapemeal. FEMS Microbiol Lett. 1994;119:83-8.

56. Mejía-Garibay B, Palou E, López-Malo A. Composition, diffusion, and antifungal activity of black mustard (Brassica nigra) essential oil when applied by direct addition or vapor phase contact. J Food Prot. 2015;78:843-8.

57. Barak E, Edgington LV. Cross-resistance of Botrytis cinerea to captan, thiram, chlorothalonil, and related fungicides. Can J Plant Pathol. 1984;6:318-20.

58. Gallori E, Casalone E, Nocita O, Turbanti L, Polsinelli M. Isolation and Characterization of a Glutathione-overproducing. Captan-resistant Mutant of Azospirillum brasilense Microbiology. 1988;134:3173-8.

59. Lundberg DS, Lebeis SL, Paredes SH, Yourstone S, Gehring J, Malfatti S, et al. Defining the core Arabidopsis thaliana root microbiome. Nature. 2012;487:86-90.

60. Schulz B, Boyle C, Draeger S, Römmert A-K, Krohn K. Endophytic fungi: a source of novel biologically active secondary metabolites. Mycol Res. 2002; 106:996-1004.

61. Lazzeri L, Malaguti L, Cinti S, Ugolini L, De Nicola GR, Bagatta M, et al. The Brassicaceae biofumigation system for plant cultivation and defence. An Italian twenty-year experience of study and application. 2013.

62. Drobnica L, Zemanova M, Nemec P, Antoš K, Kristian P, Štullerová A, et al. Antifungal activity of isothiocyanates and related compounds I. Naturally occurring isothiocyanates and their analogues. Appl Microbiol. 1967;15:701-9.

\section{Ready to submit your research? Choose BMC and benefit from:}

- fast, convenient online submission

- thorough peer review by experienced researchers in your field

- rapid publication on acceptance

- support for research data, including large and complex data types

- gold Open Access which fosters wider collaboration and increased citations - maximum visibility for your research: over $100 \mathrm{M}$ website views per year

At BMC, research is always in progress.

Learn more biomedcentral.com/submissions 